\title{
«Tu sei acqua». Dialogo e dissolvenza tra Roni Horn e Anne Carson
}

\author{
Antonella AnEDDA AngIOY \\ Università della Svizzera italiana
}

\begin{abstract}
Il titolo dell'intervento è una variazione sul titolo You are the Weather dell'artista Roni Horn, realizzato in Islanda: il viso di una giovane donna veniva fotografato durante diverse condizioni meteorologiche mentre si bagnava nelle sorgenti dell'isola. La ricerca di Horn sull'identità, sul suo comporsi e decomporsi, sulla meteorologia delle nostre emozioni trova un'interlocutrice nella scrittrice e poeta di origini canadesi Anne Carson.
\end{abstract}

Keywords: acqua, identità, conoscenza, dissoluzione, Anne Carson, Roni Horn.

Nel I840 Hippolyte Bayard scelse di fotografare se stesso come annegato, intitolando l'opera Autoportrait en noyé. In un bagno di luce paglierina il corpo esposto sembra già corroso. Il corpo immerso nell'acqua si decompone, perde i suoi tratti, i confini, diventa un'altra cosa. C'è un momento? Qual è la relazione tra acqua e identità?

Domande aperte anzi domande fluide. Carson e Horn s'interrogano sulla natura liquida del linguaggio, entrambe fanno dell'acqua un oggetto di riflessione, un corrispettivo oggettivo.

Se Carson disegna vulcani, Horn non è solo artista visiva. Il suo monologo Saying the water in 6I minuti di flusso di parole riflette sull'acqua, parla con l'acqua seguendola con lo sguardo, facendo oscillare i pensieri. L'acqua è androgina e ti solleva dal peso di ogni definizione, ti rende invisibile e ti solleva dalla necessità dello sguardo altrui:

When you talk of the water, are you talking of yourself, or of the weather? The river surrounds you and takes you away. Dark water makes you invisible, while also relieving you from the demands of sight. Thinking about water, is thinking about the future. And importantly, water is sexy. Because it is powerful, vulnerable, energetic, fragile. Near it. Immersed in it. Deeper into it. Washing all over me (Horn 20or: [trascrizione dell'autrice]).

Quando parliamo dell'acqua stiamo parlando di noi stessi o del tempo (atmosferico)? I fiumi ci circondano e ci trascinano via, l'acqua scura ci rende invisibili, ci solleva dal peso di essere visti. L'acqua è futuro, l'acqua è sensualità perché è potente, vulnerabile, è energia e fragilità.

Immersione, sprofondamento. L'acqua, si potrebbe aggiungere con le parole di Elizabeth Bishop nei versi finali della poesia At the Fishhouses, è 
come la conoscenza: scura, salata, limpida, libera, se vi immergessimo il polso, proveremmo dolore e bruciore, se l'assaggiassimo sentiremmo l'amaro. Se la osserviamo constatiamo che in movimento, esattamente come la conoscenza, è esistita, esiste, esisterà.

Nel 1999 Roni Horn realizza il libro di immagini e testi Still water, acque semplici, acque ferme, immobili, nature morte dell'acqua? Il corpo immerso nell'acqua si decompone, perde i suoi tratti, i confini, diventa un'altra cosa.

Quando è che una cosa diventa un'altra?

Le immagini di Still water hanno come oggetto le acque del Tamigi. Ogni foto è stata scattata a distanza ravvicinata e mostra solo il fiume. Niente sponde, niente presenze umane, solo le acque catturate dallo sguardo di notte, all'alba, nel pomeriggio, con tempi atmosferici diversi. Le acque sono ora trasparenti, ora oleose, ora nere e dense, ora solide e grigie, ora verde chiaro o verde giallo. A volte somigliano all'interno di una piscina, altre al grasso della balena. Ogni foto è disseminata da una galassia di numeri che formano una schiuma fluttuante sulla superficie dell'acqua, una galassia di costellazioni. In realtà i numeri rimandano o a note a fine di pagina, dove informazioni tecnico-scientifiche sugli annegati si intrecciavano a citazioni letterarie da Emily Dickinson, Joseph Conrad, John Ruskin e Paul Celan, suicida per acqua, naturalmente Anne Carson. Il fiume è un solvente, dissolve quella che chiamiamo la nostra identità. Di una giovane francese restavano solo vestiti e borsetta.

A partire dal 1990 fino al 2000 nei dieci libri intitolati To Place, Horn indaga il suo rapporto con l'Islanda, dove viaggia in motocicletta a partire dal I970. Vive a lungo in un faro. L'Islanda è una terra di vulcani, di acque mobili, di solitudini, di mutamenti. In To Place, Horn riutilizza molte immagini della serie di You are the Weather (I994-I996) in cui un viso femminile viene fotografato in tempi (meteorologici) diversi mentre si immerge nelle molte pozze di acqua calda che costellano l'Islanda. Horn lascia che il vapore di una nube o la luce del sole attraversi e modifichi il corpo che fotografa. Un viso è ombrato o illuminato, sereno o imbronciato. I termini usati dalla meteorologia possono adattarsi agli stati d'animo. Noi siamo il tempo che passa e il tempo atmosferico.

Parafrasando i versi di Wallace Stevens, uno dei poeti citati da Horn, in Thirteen Ways of Looking at a Blackbird: se ci sono molti modi di osservare un merlo, ci sono molti modi di guardare l'acqua.

$\mathrm{Nel} 2007$ Horn realizza il suo più concreto vocativo all'acqua: la Library of Water sulla costa islandese a Akureyri. L'installazione, nel centro della città di Stykkishòlmur, in una vecchia biblioteca, è fatta di colonne di vetro trasparente che contengono acque provenienti da ventiquattro differenti ghiacciai che con i cambiamenti climatici si stanno sciogliendo. Chi guarda vede i propri lineamenti perdersi in un'acqua continuamente modificata 
dalla luce. Non possiamo parlare dell'acqua senza parlare di noi stessi, dice Horn. L'acqua, le fa eco Carson, è qualcosa che non si può trattenere, l'acqua insegna la sete, aveva notato Emily Dickinson. Diversi tipi d'acqua indicano diverse seti, desiderio, conoscenza, dissoluzione, liquido amniotico e liquame.

Diving, il testo che apre la sezione intitolata The Anthropology of Water in Plainwater pubblicato da Carson nel 1995 mostra il dissolvimento dei nostri legami, la nostra impotenza a trattenere chi amiamo, esattamente come è impossibile trattenere l'acqua tra le dita, ma è anche un'indagine su ciò che il nostro cervello fatto anche d'acqua pensa dell'acqua: acqua, abbandono. L'acqua con cui le figlie di Danao sono costrette a riempire il setaccio sconfina nel racconto sul padre colpito da Alzheimer. La malattia mentale scioglie i legami, dissolve i nessi, le parole perdono il loro significato, il lessico deraglia proprio là dove sembra normale, come per esempio l'affermazione del padre che definisce una cosa «as obvious as a door in water» (Carson I995: II9). La pazzia non è separata dalla salute mentale. Non ci sono porte tra loro, ma un flusso continuo. Il cervello in acqua liquida in una sola battuta l'illusione dell'identità della figlia quando indicandola chiede alla moglie: 'Chi è quella donna?' («Who is that woman?»; I88). Qui l'acqua è perdita dei propri margini, dissoluzione, decreazione. La natura dell'acqua è limpida ma ambigua, lo sappiamo, è sete di pensiero. La sua natura è il movimento e la libertà. Eppure nota Carson in Kinds of Water: 'Certi tipi d'acqua ci annegano' («kinds of water drown us»; 130) e possono essere annunciati da un’immagine apparentemente serena come quella di una cascata dove si vede un'ombra che sembra un cane mor to ed è un cane morto, annegato. Le forme trasmutano, le acque diventano pioggia, mare, fiumi, lacrime. Quando è che una cosa diventa un'altra e come avviene? Poesia, racconto, biografia, io, tu, loro, esistono davvero? I nomi hanno senso, i pronomi sono reali o sono solo scritti sull'acqua? Water Margins: An Essay on Swimming by my Brother (ultimo testo di Plainwater) riflette sulla fraternità e sulla natura liquida di questo legame. Il racconto viene accennato ma sgocciola via. C'è un fratello caratteriale e una sorella compassionevole. Nel lago incastonato nella terra l'acqua non fluisce disordinatamente ma viene trattenuta, sprofonda senza margini: uomo e donna, gruppi, saggezza, antiche religioni, amore, impossibilità d'amore, tutto perde fisionomia. L'acqua rode l'identità. Chi sei tu, chi sono io? Pensiamo alle foto di Roni Horn in cui non ci sono barriere tra femminile e maschile, in cui il viso (di lei?) trabocca in quello (di lui?) e viceversa per riversarsi nel paesaggio. Il nuotatore nuota, ci immergiamo con lui, lo seguiamo, i suoni sono quelli delle bracciate, delle onde, durante l'immersione affiorano brandelli di una vita alla deriva. Il ritmo è quello delle braccia e delle gambe, del respiro e dell'affanno, del fiato che viene trattenuto e poi esce, si placa, riprende. I suoni si affievoliscono, i sogni si moltiplicano, 
il mondo compare, dilaga, sparisce. I richiami della vita con le sue illusioni sembrano chiamare inutilmente dalla sponda dove il buon senso non ha più senso per chi si è spinto troppo al largo. Chi ama nuotare sa che una delle sensazioni più esaltanti è quella di perdere la nozione di se stessi nell'acqua, che occorre abbandonarsi per non affondare, se il corpo sente di esserci si fa pesante. Per non affogare bisogna saper fare il morto. Nel 20I0, il fratello di Carson protagonista senza nome di Diving, muore a Copenhagen. Nello stesso anno esce Nox, il libro: una teca grigia con un bordo bianco (il latino nox slitta in box, 'scatola') diluisce il carme Ior di Catullo, lo disfa verso dopo verso facendo navigare le parole che si immergono nel lutto, riaffiorano, sprofondano di nuovo. Dove finisce la poesia? Dove iniziamo noi? Sei anni dopo Carson pubblica Float, ancora un richiamo all'acqua, piccoli libri sottili, smembrabili, leggibili in momenti diversi. Un'installazione di lettura. Nella vasca-scatola trasparente le parole fluttuano, i concetti si possono comporre e scomporre, leggere separatamente, di nuovo i rimandi letterari non hanno bordi. Un'idea di acqua che ancora una volta dissolve e interroga il linguaggio.

Nel 2006 Horn aveva realizzato una serie di sculture (Glass) dove luce e vetro creavano l'impossibile: la densità tattile dell'acqua. Glass, Irony and God è il titolo di un libro di Carson pubblicato nel 1992, un capolavoro liquido che scorre dalla poesia alla teologia, toccando luoghi e pensieri diversi: Canada e Roma, Emily Brontë e Aristotele. 'L'acqua è qualcosa che non puoi tenere' aveva scritto Carson in Plainwater (1995: 274). Siamo sicuri? Forse le parole non possono, ma l'arte visiva sì. Colonne, cilindri, materiali che si possono toccare. Qualcosa di solido? Forse.

\section{Bibliografia}

Bishop, Elizabeth, The Complete Poems, New York, Straus and Giroux, 1980. Carson, Anne, Plainwater, New York, Knopf, 1995.

-. Nox, New York, New Directions, 2010.

-. Float, New York, Knopf, 2016.

Horn, Roni, Saying Water [Cd], New York, Dia Foundation, 200 .

-. To Place [1990], Göttingen, Steidl, 2004.

-.You are the Weather [1997], Göttingen, Ed. De Salvo, 2009.

-. Still Water, Göttingen, Ed. De Salvo, 1999.

Stevens, Wallace, The Collected Poems, New York, Knopf, 1982. 